\title{
Characteristics of Auditory Stereocilia in the Apical Turn of the Echolocating Bats by Scanning Electron Microscopy
}

\author{
Jinyong Kim ${ }^{1}$, Yongwook Jung ${ }^{1,2, *}$ \\ ${ }^{1}$ Laborotory of Electron Microscopy, ${ }^{2}$ Department of Anatomy, Dongguk University College of Medicine, Gyeongju 780-714, Korea
}

*Correspondence to:

Jung $Y$,

Tel: $+82-54-770-2404$

Fax: $+82-54-770-2447$

E-mail: jungyw@dongguk.ac.kr

Received March 10, 2014

Revised March 18, 2014

Accepted March 24, 2014

\begin{abstract}
The auditory system of the Korean greater horseshoe bat (Rhinolophus ferrumequinum korai, RFK) is adapted to its own echolocation signal, which consist of constant frequency (CF) element and frequency modulated (FM) element. In contrast, the Japanese longfingered bat (Miniopterus schreibersii fuliginosus, MSF) emits FM signals. In the present study, the characteristics of stereocilia in RFK (a CF/FM bat) and MSF (a FM bat) were studied in the apical turn of the cochlea where the lower frequencies are transduced. Stereocilia lengths and numbers were quantitatively measured in $R F K$ by scanning electron microscopy and compared with those of MSF. Each inner hair cells (IHCs) of $R F K$ possessed three rows of stereocilia, whereas MSF possessed five rows of stereocilia. Gradients in stereocilia lengths and numbers of stereocilia of the IHCs of $R F K$ were found to be less pronounced and fewer, respectively, than those of MSF. Each outer hair cells (OHCs) possessed three rows of stereocilia in both species. OHCs stereocilia in RFK that distinguished it from $M S F$ were a shorter length and a greater number of stereocilia. These features suggest that the apical cochleas of RFK are adapted for the processing of higher frequency echolocation calls rather than that of MSF.
\end{abstract}

Key Words: Echolocating bats, Scanning electron microscopy, Stereocilia

\section{INTRODUCTION}

As insectivorous microchiropteran bats, Korean greater horseshoe bats use echolocation to orient within the environment and prey on flying insects (Chung et al., 2010). Rhinolophus ferrumequinum sp. widely inhabits Europe, China, and Korea. Recently populations have been declining in Europe, whereas their number is growing in the Korean peninsula (Csorba et al., 2003). However, few research studies have been conducted on the Korean greater horseshoe bats in the Republic of Korea.

Echolocation is a type of biological sonar that is used by several types of echolocating animals. These animals emit calls into the environment, listen for returning echoes, and use these to locate and identify objects. The echolocation calls of the Korean greater horseshoe bat ( $R$. ferrumequinum korai, RFK) consist of long constant frequency (CF) element, which is invariably terminated by a brief downward frequency modulated (FM) element (49 kHz) (Chung et al., 2010). The CF component is used to detect wing-beating insects in dense foliage, and its frequency is species-specific $(R$. ferrumequinum, $83 \mathrm{kHz}$; Rhinolophus rouxi, $78 \mathrm{kHz}$ ) (Vater \& Lenoir, 1992). In contrast, the Japanese long-fingered bat, Miniopterus schreibersii fuliginosus (MSF), while foraging on the wing, emits sequences of brief, downward-FM signals $(80 \sim 20 \mathrm{kHz})$ with a low dominant frequency of $44 \mathrm{kHz}$ when approaching and catching prey (Jones \& Teeling, 2006; Liang et al., 2011).

The mammalian cochlea performs a frequency analysis of acoustic signals containing low to high frequency tones by systematically processing these signals using hair cells located in the apical to basal region of the cochlea, respectively. The

This work was supported by the Dongguk Research Fund.

@ This is an open-access article distributed under the terms of the Creative Commons Attribution Non-Commercial License (http://creativecommons.org/licenses/by-nc/3.0) which permits unrestricted noncommercial use, distribution, and reproduction in any medium, provided the original work is properly cited.

Copyrights @ 2014 by Korean Society of Microscopy 
basis of this frequency map is primarily attributed to the natural vibrating frequency of the basilar membrane along the cochlear duct (Bekesy, 1960). Other factors, however, including the properties of the tectorial membrane, the lengths and numbers of hair cell stereocilia (Steel, 1983) and the stiffness variations of stereocilia (Strelioff \& Flock, 1984) may also play a role in determining the frequency selectivity. In a recent study, it was proposed that the micromechanical properties of hair cell stereocilia play important roles in the detection of frequency changes and in electro-mechanical transduction (Lim, 1986). The lengths and numbers of auditory stereocilia have been studied in echolocating bats in the apical, middle, and basal turns (Yao et al., 2007). In addition, relations between stereocilia lengths and quantities and hearing at different hair cell locations have been studied in the bird cochlea (Tilney \& Saunders, 1983). However, the precise roles played by stereocilia length and number are not fully understood as they vary by cochlea segment and between species.

Thus, in the present study, we examined the characteristics of stereocilia at the fifth half-turn $(8.1 \sim 8.5 \mathrm{~mm}$ from the basal end) of cochlea in two different types of echolocating bats by scanning electron microscopy (SEM). Bruns \& Schmieszek (1980) demonstrated that the apical region from $8 \mathrm{~mm}$ to the apex represented frequencies lower than $40 \mathrm{kHz}$ in the greater horseshoe bat ( $R$. ferrumequinum). Apical turns have received less research attention than the basal turns of CF/FM and FM bats, which process high frequency echolocation signals. Furthermore, no investigation has been conducted on the stereocilia of the apical turn of RFK (a CF/FM bat) or of MSF (a FM bat), nor has any comparative study been undertaken on the auditory stereocilia of CF/FM and FM bats.

\section{MATERIALS AND METHODS}

\section{Animals}

Animals were captured in an abandoned mine in the
Tongyeong area (Gyeongsangnam-do, South Korea) between March and April 2013. Experimental procedures were reviewed and approved by the Animal Care and Use Committee of Dongguk University, and animal care and use were in accord with the guidelines issued by the National Institute of Health (Bethesda, MD, USA). Three cochleae from both species were used in the study.

\section{Scanning Electron Microscopy}

Bats were decapitated under ether anesthesia and cochleae were quickly removed from temporal bones and perfused in a fixative containing $2.5 \%$ glutaraldehyde in $0.1 \mathrm{~mol} / \mathrm{L}$ sodium cacodylate buffer ( $\mathrm{pH}$ 7.2). Bony otic capsules were removed using a forceps under a dissecting microscope to expose labyrinths. Specimens were fixed in the same fixative at $4^{\circ} \mathrm{C}$, rinsed in $0.1 \mathrm{~mol} / \mathrm{L}$ sodium cacodylate buffer, postfixed in $\mathrm{OsO}_{4}$ for 2 hours, dehydrated in an increasing alcohol series (50\% 100\%), and soaked overnight in isoamyl-acetate. Cochleae were then critical-point dried using liquid $\mathrm{CO}_{2}$ (HCP-2; Hitachi, Japan) and gold sputtered (E-100; Hitachi). Specimens were examined using a field emission SEM (S-4200; Hitachi). Each fifty inner hair cells (IHCs) and outer hair cells (OHCs) from RFK and MSF were examined at the fifth halfturn (8.1 8.5 mm from the basal end) of cochleas (a distance of between 69\% 66\% (Fig. 1A, rectangle) and 69\% 71\% (Fig. $1 \mathrm{~B}$, rectangle), respectively, from the basal end.

\section{Statistical Analysis}

To provide accurate measures of stereocilia length (from root to apex), prone stereocilia in SEM photographs were erected and stereocilia length values were obtained using SEM software (USB20 Image Software; Korea Research Institute of Standards and Science, Korea). Photomicrographs of IHCs stereocilia at different positions along sensory epithelium were evaluated to determine numbers per row, which provides a good measure of numbers of stereocilia per cell, despite vague borders between hair cells in SEM images. Micrographs
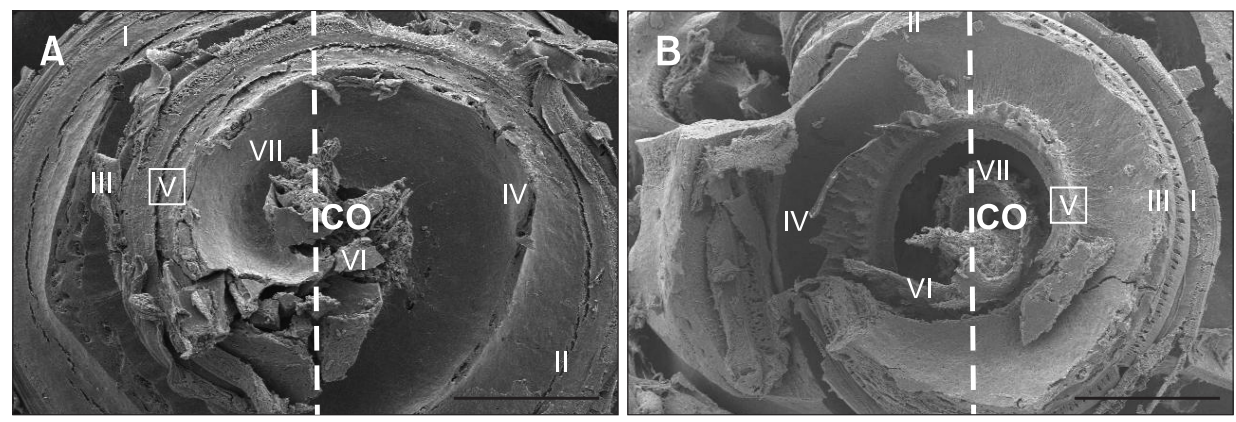

Fig. 1. Scanning electron micrographs of the cochleas of RFK (a CF/FM echolocater) (A) and MSF (a FM echolocater) (B). Individual half-turns (1st to 7th half turns) are numbered starting from basal ends. The vertical dotted lines divide cochleas into 7 half-turns. The stereocilia of inner hair cells and outer hair cells of RFK and MSF were examined at the fifth half-turn (rectangle). CO, cochleas; RFK, Rhinolophus ferrumequinum korai; $\mathrm{CF}$, constant frequency; FM, frequency modulated; MSF, Miniopterus schreibersii fuliginosus. Scale bars=500 $\mu \mathrm{m}$. 
were taken at $\times 3,000$ and enlarged to provide an effective magnification of at least $\times 20,000$. Numbers of IHCs stereocilia per row were counted using the axis of the helicotrema at the 12 o'clock position, which was used as a reference point. Initially, stereocilia were counted $0.5 \mathrm{~mm}$ to the left and right of the reference point, and then $1 \mathrm{~mm}$ to the left and right around the same reference point. Numbers of OHCs stereocilia were determined by counting the total numbers of stereocilia bundles. The opening angles of stereocilia bundles of OHCs were measured using a protractor. Data were analyzed using Excel software (Microsoft Corp., USA), and results are expressed as mean \pm standard deviation. Groups were compared using unpaired $\mathrm{t}$-test. $\mathrm{p}$-values of $<0.05$ were considered significant.

\section{RESULTS}

The mean diameters of cochleas from RFK and MSF were 2.2 and $2.0 \mathrm{~mm}$, respectively, total cochlear lengths were 12.3 and $11.8 \mathrm{~mm}$ and cochleas had 3.5 and 3.4 turns, respectively. $R F K$ had a larger cochlear diameter and length than MSF. The cochlea in RFK and MSF were found to possess three and a half turns or 7 half-turns by bisecting cochleas vertically, as shown in Fig. 1.

Scanning electron micrographs of IHCs and OHCs at the apical turn of the organ of Corti of RFK and MSF are shown in Fig. 2. At the apical turn in both species, there were three rows of OHCs and one row of IHCs, which concurs with the description of $R$. rouxi (a related bat species) by Vater \& Lenoir (1992). IHCs were observed to adjoin inner phalangeal cells and one row of inner pillar cells. OHCs were arranged in a mosaic pattern comprised of alternating junctions with one row of outer pillar cells (OPs) and three rows of Deiters 1 (D1), Deiters 2 (D2), and Deiters 3 (D3) cells (Fig. 2). IHCSs of stereocilia in RFK had regular, closely spaced stereocilia, whereas those of MSF had irregularly separated stereocilia bundles (Fig. 2). The two species had similar OHCs spacing patterns. In RFK, the innermost row (Fig. 2A, '(1)') and the middle row (Fig. 2A, '(2)') were distinctly separated by OPs. In contrast, in $R F K$, there was little space between the middle and outermost rows (Fig. 2A, '(3)') of OHCs.

\section{Inner Hair Cells Stereocilia in the Two Species}

In both species, IHCs stereocilia bundles were arranged linearly, which contrasted with the ' $\mathrm{W}$ ' shaped arrangement of OHCs stereocilia bundles (Fig. 2). Stereocilia in RFK were usually independently freestanding (Fig. 3A), whereas the stereocilia of $M S F$ were organized into bundles (Fig. 3B). IHCs stereocilia in the two species were tapered with club-like blunt tips (Fig. 3). In $R F K$, IHCs stereocilia decreased in length from a maximal value of $1.46 \mu \mathrm{m}$ in the outermost row (Fig. 3A, '(3)') to $0.56 \mu \mathrm{m}$ in the innermost row (Fig. 3A, '(1)'). Similarly, stereocilia lengths in MSF gradually decreased in length from a maximal of $1.56 \mu \mathrm{m}$ in the outermost row (Fig. 3B, '(5)') to $0.28 \mu \mathrm{m}$ in the innermost row (Fig. 3B, '(1)'). These results suggest that stereocilia lengths exhibit smaller gradients in RFK than in MSF. The numbers of IHCs stereocilia were greater in MSF than in RFK ( $R F K, 59$ vs. MSF, 90; $\mathrm{p}<0.05)$, because IHCs in $R F K$ possessed three rows of stereocilia, whereas MSF possessed five (Fig. 3). The lengths and numbers of IHCs stereocilia in RFK and MSF are summarized in Table 1.

\section{Outer Hair Cells Stereocilia in the Two Species}

In both species, stereocilia bundles of OHCs were arranged in a slight curve in microscopic view. There were three rows
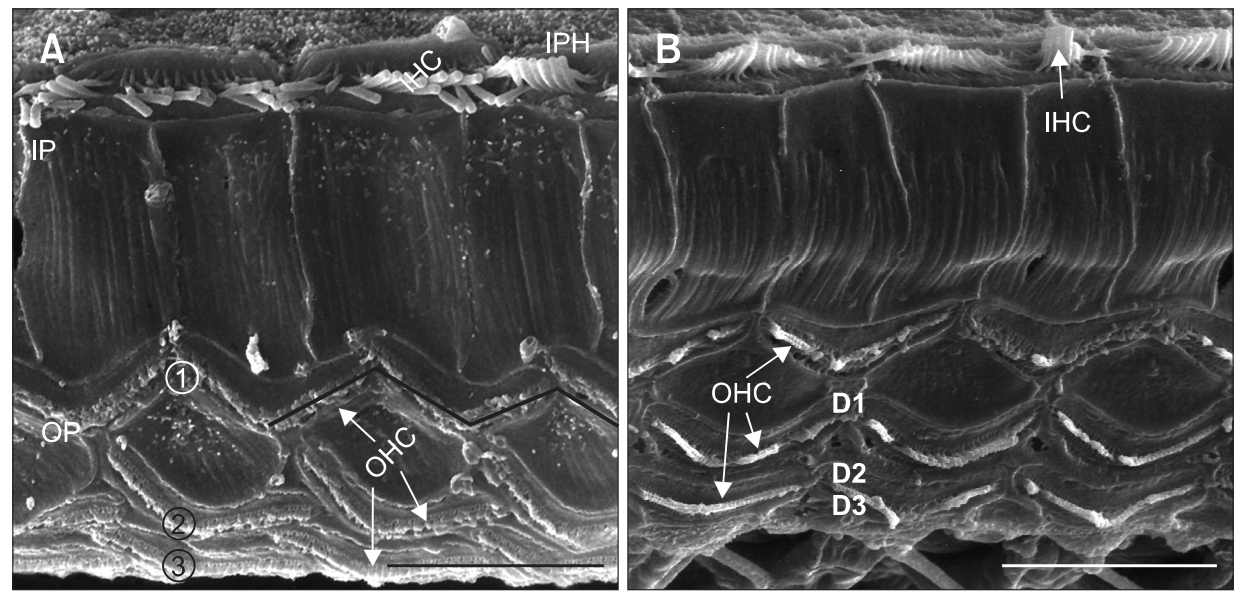

Fig. 2. Scanning electron micrographs of IHCs and OHCs at the apical turns in the cochlea of RFK (A) and MSF (B). One row of IHCs and three rows of OHCs (1), innermost row; (2), middle row; (3), outermost row) were observed in both species. The distinguishing feature of IHCs stereocilia in MSF as compared with $R F K$ was the wide spatial separation of stereocilia bundles. RFK had more regular, closely spaced freestanding stereocilia. OHCs stereocilia in both species were arranged in a 'W' manner (A). IP, inner pillar cell; IHC, inner hair cell; IPH, inner phalangeal cell; OP, outer pillar cell; OHC, outer hair cell; D1, D2, D3, three rows of deiter cells; RFK, Rhinolophus ferrumequinum korai; MSF, Miniopterus schreibersii fuliginosus. Scale bars=10 $\mu \mathrm{m}$. 

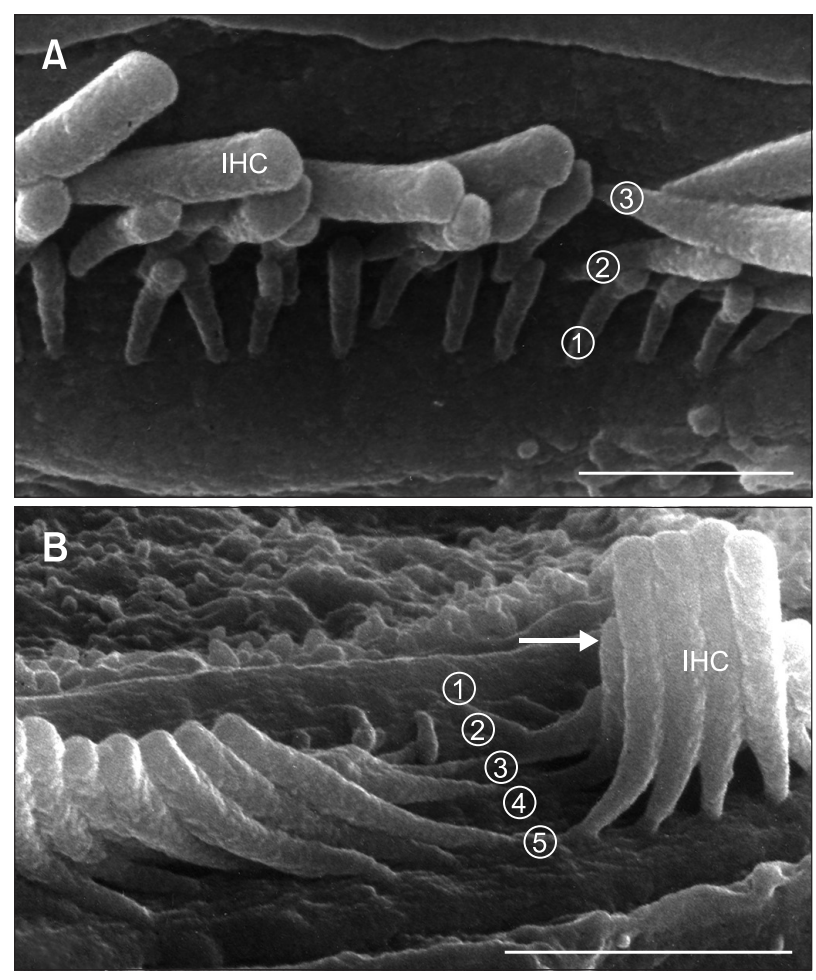

Fig. 3. Scanning electron micrographs of IHCs at the apical turn in the cochlea of RFK (A) and MSF (B). One row of IHCs possessed three rows of stereocilia in $R F K$ (1), innermost row; (2), middle row; (3), outermost row). Note the one row of IHCs with five rows of stereocilia (1), innermost row; (2), inner row; (3), middle row; (4), outer row; (5), outermost row) and the fused form of stereociliary bundles (white arrow) (B) in MSF. IHC, inner hair cell; RFK, Rhinolophus ferrumequinum korai; MSF, Miniopterus schreibersii fuliginosus. Scale bars $=1.5 \mu \mathrm{m}$.

Table 1. IHCs stereocilia lengths and numbers in the apical turn of the two bat species

\begin{tabular}{lccccc}
\hline \multirow{2}{*}{ Row } & \multicolumn{2}{c}{$\begin{array}{c}\text { Rhinolophus } \\
\text { ferrumequinum korai }\end{array}$} & & \multicolumn{2}{c}{$\begin{array}{c}\text { Miniopterus schreibersii } \\
\text { fuliginosus }\end{array}$} \\
\cline { 2 - 3 } \cline { 5 - 6 } & Length $(\mu \mathrm{m})$ & Number & & Length $(\mu \mathrm{m})$ & Number \\
\hline IHC1 (innermost) & $0.56 \pm 0.02$ & $22 \pm 0.5$ & & $0.28 \pm 0.03$ & $19 \pm 0.2$ \\
IHC2 (inner) & - & - & & $0.80 \pm 0.04$ & $18 \pm 0.2$ \\
IHC3 (middle) & $0.94 \pm 0.02$ & $19 \pm 0.6$ & & $0.93 \pm 0.02$ & $19 \pm 0.2$ \\
IHC4 (outer) & - & - & & $0.98 \pm 0.01$ & $17 \pm 0.2$ \\
IHC5 (outermost) & $1.46 \pm 0.03$ & $18 \pm 0.3$ & & $1.56 \pm 0.03$ & $17 \pm 0.4$ \\
Total & - & $59 \pm 1.5$ & & - & $* 90 \pm 0.7$ \\
\hline
\end{tabular}

Values are presented as mean \pm standard deviation. Number $=3$. IHC, inner hair cell. ${ }^{*} \mathrm{p}<0.05$.

of OHCs and each possessed three rows of stereocilia of a different length, resembling steps (Fig. 4). In RFK (a CF/FM bat), OHCs stereocilia decreased in length from a maximal value of $0.38 \mu \mathrm{m}$ in the outermost row (Fig. $4 \mathrm{~A}$, '(3)') to 0.19 $\mu \mathrm{m}$ in the innermost row (Fig. 4A, '(1)'), whereas OHCs stereocilia in MSF (a FM bat) decreased in length from a
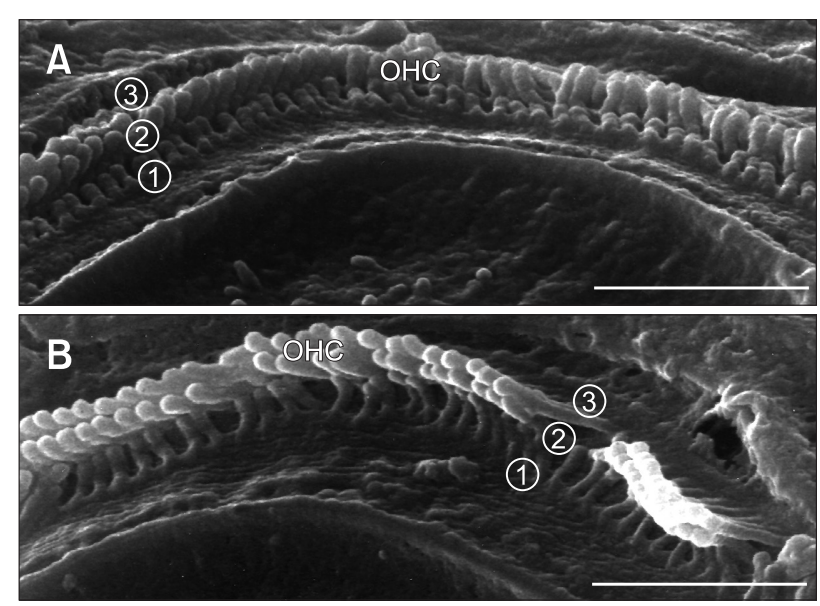

Fig. 4. Scanning electron micrographs of OHCs at the apical turn in the cochlea of RFK (A) and MSF (B). Each row of OHCs in both species possessed three rows of stereocilia (1), innermost row; (2), middle row; (3), outermost row). OHC, outer hair cell; RFK, Rhinolophus ferrumequinum korai; MSF, Miniopterus schreibersii fuliginosus. Scale bars $=1.5 \mu \mathrm{m}$.

Table 2. OHCs Stereocilia lengths and numbers in the apical turn of the two bat species

\begin{tabular}{lccccc}
\hline \multirow{2}{*}{ Row } & \multicolumn{2}{c}{$\begin{array}{c}\text { Rhinolophus } \\
\text { ferrumequinum korai }\end{array}$} & & \multicolumn{2}{c}{$\begin{array}{c}\text { Miniopterus schreibersii } \\
\text { fuliginosus }\end{array}$} \\
\cline { 2 - 3 } \cline { 5 - 6 } & Length $(\mu \mathrm{m})$ & Number & & Length $(\mu \mathrm{m})$ & Number \\
\hline OHC1 (innermost) & $0.19 \pm 0.01$ & ${ }^{*} 50 \pm 0.5$ & & ${ }^{*} 0.26 \pm 0.01$ & $38 \pm 0.7$ \\
OHC2 (middle) & $0.28 \pm 0.01$ & ${ }^{*} 58 \pm 0.4$ & & $* 0.47 \pm 0.01$ & $43 \pm 0.5$ \\
OHC3 (outermost) & $0.38 \pm 0.03$ & ${ }^{*} 64 \pm 0.3$ & & $* 0.56 \pm 0.02$ & $45 \pm 0.3$ \\
\hline
\end{tabular}

Values are presented as mean \pm standard deviation. Number $=3$. OHC, outer hair cells. ${ }^{*} \mathrm{p}<0.05$.

maximal value of $0.56 \mu \mathrm{m}$ in the outermost row (Fig. 4B, (3)') to $0.26 \mu \mathrm{m}$ in the innermost row (Fig. 4B, '(1)'). OHCs stereocilia lengths in $R F K$ were shorter than in $M S F$ for all three rows $(\mathrm{p}<0.05)$. Opening angles and OHCs stereocilia numbers were found to show irregularities. The opening angle of stereocilia bundles in RFK increased from the innermost row $\left(124^{\circ}\right)$ to the outermost row $\left(148^{\circ}\right)$ (Fig. 4A, (1) (3)'), and numbers of stereocilia from the innermost to the outermost row increased from 50 to 64 depending on opening angle (Fig. 4A, '(1) (3)'). In MSF, the opening angles of stereocilia bundles were smaller; opening angles and numbers of stereocilia from the innermost row to the outermost row were $117^{\circ}$ to $121^{\circ}$ and 38 to 45 , respectively (Fig. $4 \mathrm{~B}$, (1) (3)'). The number of OHCs stereocilia in RFK were greater than in MSF for all three rows $(\mathrm{p}<0.05)$. These results suggest that the lengths and numbers of OHCs stereocilia in $R F K$ are shorter and more numerous than those of MSF. The lengths and numbers of OHCs stereocilia of RFK and MSF are summarized in Table 2. 


\section{DISCUSSION}

Studies on the audition systems of echolocating bats have demonstrated that they are intricately adapted to the requirements of species-specific acoustic foraging strategies (Neuweiler, 2003). For instance, $R$. rouxi, which pursue flying insects in or around dense vegetation, have evolved a sophisticated cochlear fovea and neuronal specializations that allow the selective detection of wing-beating targets among echo-clutter. In this bat, the morphological organizations of cochlear hair cells are probably related to the tuning capacities and sensitivities of receptor organs (Vater \& Lenoir, 1992). The Korean greater horseshoe bat in the Republic of Korea uses $\mathrm{CF}$ and FM components during echolocation. In the present study, we analyzed and compared the basic and characteristic stereocilia structures of RFK and MSF which utilize CF/FM and FM echolocation patterns, respectively. In particular, we examined the fifth half-turn $(8.1 \sim 8.5 \mathrm{~mm}$ from the basal end) of the cochlea which corresponds with distances of between $69 \%$ and $66 \%(R F K)$ and $69 \%$ and $71 \%$ $(M S F)$ from the basal end, respectively. Based on cochlear innervation features, such as, densities of spiral ganglion and of inner and outer receptors, the cochlea of greater horseshoe bats can be subdivided into three regions: (1) the basal region $1.3 \sim 5.4 \mathrm{~mm}(8 \% \sim 33 \%)$ from the basal end where CF segments of echolocation signals are detected (around $83 \mathrm{kHz})$; (2) the middle region 5.4 8 $\mathrm{mm}(33 \% \sim 50 \%)$ from the basal end where FM segments of echolocation signals are detected $(40 \sim 80 \mathrm{kHz})$; and (3) the apical region from 8 $\mathrm{mm}$ to the apex $(16 \mathrm{~mm})$ where frequencies lower than 40 $\mathrm{kHz}$ are detected (Bruns \& Schmieszek, 1980). In another study, Tadardia brasiliensis (a FM bat), the most sensitive and sharpest tuning region between 35\% and 80\% distant from the base was found to detect frequencies between 30 and 12 $\mathrm{kHz}$ (Vater \& Siefer, 1995). These results suggest that apical turn of RFK and MSF processes lower frequencies than that of basal turn.

\section{Inner Hair Cells Stereocilia in the Apical Turns of Rhinolophus ferrumequinum korai and Miniopterus schreibersii fuliginosus}

In the present study, IHCs stereocilia length ranges in $R F K$ (a CF/FM bat) and MSF (a FM bat) were 1.46 0.56 $\mu \mathrm{m}$ and 1.56 0.28 $\mu \mathrm{m}$, respectively. The small range of IHCs stereocilia length found in the apical turn of $R F K$ than in $M S F$ is believed to be due to adaption to narrow frequency bands. In contrast, the broad range of stereocilia length in the IHCs of MSF may be compensated for an extended hearing range than in RFK. IHCs stereocilia in the basal turn of the cat are $2.3 \mu \mathrm{m}$ long in the $38 \mathrm{kHz}$ region and $7.1 \mu \mathrm{m}$ long in the apical turn (the $0.175 \mathrm{kHz}$ region) (Frishkopf \& DeRosier, 1983). Moreover, IHCs stereocilia length in the basal turn of the mouse is $3.5 \mu \mathrm{m}$ in the $20 \mathrm{kHz}$ region (Lim, 1986). Shorter stereocilia lengths of IHCs in the apical turns of RFK and MSF than in non-echolocating mammals may provide an explanation for their higher hearing frequency ranges. Furthermore, these results indicate that the hearing range of echolocating bats does not reach as far into the low frequency ranges as in the non-echolocating mammals.

In the present study, the numbers of IHCs stereocilia in MSF were larger than in RFK (MSF, 90 vs. RFK, 59). It is well known that IHCs are the main sensory cells in the auditory system and their activities are relayed to hearing centers in the brain. On the other hand, OHCs contribute importantly to the sensitivity and frequency resolution provided by cochlear mechanics, but only indirectly to auditory perception (Dallos et al., 2008). These ideas are consistent with known differences between organizations of the two receptor cell populations (IHCs and OHCs) at the ultrastructural (Lim, 1986) and in terms of innervation density (Pujol \& Lenoir, 1986), the coupling of stereocilia to the tectorial membrane (Lim, 1986), and motile properties (Brownell et al., 1985; Zenner, 1986; Ashmore, 1987). If transduction channels depend on the number of stereocilia (Osborne et al., 1984; Hudspeth, 1985), then the IHCs of MSF have more transduction sites than those of $R F K$, and hence, probably improve the sensitivity echolocation perception. This suggests that the cochleas of $M S F$, a FM bat, are sensitive to a lower range of frequencies than RFK, CF/FM bat. The expanded frequency range of $T$. brasiliensis (a FM bat) has a frequency best hearing band at $21 \sim 27 \mathrm{kHz}$ (Pollak et al., 1978), which according to Simmons \& Stein (1980) corresponds to the lower frequency end of FM echolocation signals.

\section{Outer Hair Cells Stereocilia in the Apical Turns of Rhinolophus ferrumequinum korai and Miniopterus schreibersii fuliginosus}

Supernumerous OHCs and missing OHCs are typical features of the cochlear apex in non-echolocating mammals (Lenoir et al., 1987), but were not observed in the apical cochlea of RFK or MSF in the present study. The three rows of OHCs stereocilia bundles play an important role in determining cochlear frequency response characteristics (Strelioff et al., 1985). In the present study, stereocilia lengths at the apical turn in both species decreased gradually from the outermost to innermost row. In non-echolocating mammals, such as, the guinea pig and chinchilla, clear gradations were also observed in middle and apical cochleas (Wright, 1984; Lim, 1986). Furthermore, this gradation has also been reported in the middle and apical turns of the echolocating bats, R. rouxi and Hipposidero bicolor (Vater \& Lenoir, 1992). Therefore, gradation of OHCs stereocilia lengths may be a common anatomic feature at the apical and middle turns of echolocating and non-echolocating mammals. 
The stereocilia lengths in the innermost to outermost rows in the apical turn of RFK (with FM values of $49 \mathrm{kHz}$ ) were 0.19 to $0.38 \mu \mathrm{m}$, which are shorter than the reported values of Pteronotus parnellii another CF/FM echolocating bat (CF value of $61 \mathrm{kHz}$ and stereocilia lengths of $0.7 \sim 0.8 \mu \mathrm{m}$ ) (Suga et al., 2003). In the present study, stereocilia lengths of the innermost to outermost rows in MSF (with FM values of $80 \sim 20 \mathrm{kHz}$ ) were 0.26 to $0.56 \mu \mathrm{m}$. T. brasiliensis employs a broadband system (Vater \& Siefer, 1995), and has a frequency range extending from 40 to $75 \mathrm{kHz}$ and a stereocilia length range of $0.8 \sim 1.0 \mu \mathrm{m}$. These results suggest that the lengths of stereocilia in echolocating bats depend on hearing frequency and species. In particular, longer OHCs stereocilia in the apical turn of the cochlea in MSF implies that this feature is used for lower resonance frequencies than in $R F K$. In contrast, the shorter stereocilia in $R F K$ probably represent an adaptation to high frequency hearing. Hudspeth \& Corey (1977) also demonstrated that shorter OHCs stereocilia in vertebrate hair cells represent a more delicate response to resonant motion when sound waves enter the ear. Therefore, differences between OHCs stereocilia lengths in RFK and MSF appear to be closely related to specialized hearing frequency ranges and susceptibilities to motions.

In the chinchilla cochlea, a widening of the opening angle of stereocilia bundles is accompanied by an increase in the number of OHCs stereocilia (Lim, 1986). In the cochleas of $R F K$, the opening angles of stereocilia bundles from the innermost to the outermost row and numbers of stereocilia per row were found to be greater than at corresponding positions in $M S F\left(R F K, 124^{\circ} \sim 148^{\circ}\right.$ and $50 \sim 64$ vs. $M S F$, $117^{\circ} \sim 121^{\circ}$ and $38 \sim 45$ ). Wide opening angles of stereocilia bundles appear to be correlated with the processing of high frequencies (Vater \& Lenoir, 1992). In R. rouxi, opening angles and numbers of OHCs stereocilia varied from $90^{\circ}$ and
40 to $130^{\circ}$ and 63 , respectively, from the apical to the basal coil. A similar observation was made in the chinchilla, in which the variation ranged from $60^{\circ}$ and 22 to $120^{\circ}$ and 35 , respectively, from the low- to the high-frequency region (Vater \& Lenoir, 1992). Thus, we would expect that the apical turn of $R F K$ represents a higher frequency range than that of other horseshoe bats and non-echolocating mammals.

\section{CONCLUSIONS}

The length gradient and the number of IHCs stereocilia at the apical cochlea of Rhinolophus ferrumequinum korai (RFK) were found to be smaller than in Miniopterus schreibersii fuliginosus (MSF). Additionally, the lengths and numbers of OHCs stereocilia in RFK were shorter and greater in number than those observed in MSF. These features suggest that the apical cochleas of RFK (a CF/FM echolocater) are adapted for the processing of higher frequency echolocation calls whereas the apical cochleas of MSF (a FM echolocater) more sensitively process low frequency components of echolocation calls.

\section{CONFLICT OF INTEREST}

No potential conflict of interest relevant to this article was reported.

\section{ACKNOWLEDGMENTS}

The author thanks professor Jin Seo Park (Department of Anatomy, Dongguk University College of Medicine) for his scientific input and Jihan Kim (Department of Anatomy, Dongguk University College of Medicine) for technical assistance.

\section{REFERENCES}

Ashmore J F (1987) A fast motile response in guinea-pig outer hair cells: the cellular basis of the cochlear amplifier. J. Physiol. 388, 323-347.

Bekesy A G (1960) Experiments in Hearing (McGraw-Hill Book Co., New York, Toronto, London).

Brownell W E, Bader C R, and Brand D (1985) Evoked mechanical responses of isolated cochlear hair cells. Science 227, 194-196.

Bruns V and Schmieszek E (1980) Cochlear innervation in the greater horseshoe bat: demonstration of an acoustic fovea. Hearing Res. 3, 27-43.

Chung C U, Han S H, and Kim S C (2010) General patterns in echolocation call of greater horseshoe bat Rhinolophus ferrumequinum, Japanese Pipistrelle abramus and large-footed bat Myotis macrodactylus in Korea. J. Enivronmental. Science 19, 61-68.

Csorba G P, Ujhelyi P, and Thomas N (2003) Horseshoe Bats of the World
(Alana Books, Shropshire, England).

Dallos P, Wu X, and Cheatham M A (2008) Prestin-based outer hair cell motility is necessary for mammalian cochlear amplification. Neuron 227, 333-339.

Frishkopf L S and DeRosier D J (1983) Mechanical tuning of free-standing stereociliary bundles and frequency analysis in the alligator lizard cochlea. Hear Res. 12, 393-404.

Hudspeth A J (1985) The cellular basis of hearing: the biophysics of hair cells. Science 230, 745-752.

Hudspeth A J and Corey D P (1977) Sensitivity, polarity and conductance change in the response of vertebrate hair cells to controlled mechanical stimuli. Proc. Natl. Acad. Sci. USA 74, 2407-2411.

Jones $G$ and Teeling E C (2006) The evolution of echolocation in bats. Trends. Ecol. Evol. 21, 49-56. 
Lenoir M, Puel J L, and Pujol R (1987) Stereocilia and tectorial membrane development in the rat cochlea. A SEM study. Anat. Embryol. 175, 477-487.

Liang H K, Li W, and Biao Z L (2011) Dietary composition, echolocation pulses and morphological measurements of the long-fingered bat Miniopterus fuliginosus (Chiroptera: Vespertilinoninae). Zoological. Research 32, 163-167.

Lim D J (1986) Functional structure of the organ of Corti: a review. Hear Res. 22, 117-146.

Neuweiler G (2003) Evolutionary aspects of bat echolocation. Comp. Physiol. A. Neuroethol. Sens. Neural. Behav. Physiol. 189, 245-256.

Osborne M P, Comis S D, and Pickles J O (1984) Morphology and crosslinkage of stereocilia in the guinea-pig labyrinth examined without the use of osmium as a fixative. Cell Tissue Res. 237, 43-48.

Pollak G D, Marsh D S, and Bodenhamer R (1978) A single unit analysis of inferior colliculus in unanestheized bats: response patterns and spike count functions generated by constant frequency and frequency modulated sounds. J. Neurophysiol. 41, 677-691.

Pujol R and Lenoir M (1986) The four types of synapses in the organ of Corti. In: Neurobiology of Hearing: The Cochlea, eds. Altschuler R A, Bobbin R P, and Hoffman D W, pp. 161-173, (Raven Press, New York).

Simmons J A and Stein R (1980) Acoustic imaging in bat sonar: echo location signals and the evolution of echolocation. J. Comp. Neurol. 135, 61-84.

Steel K P (1983) The tectorial membrane of mammals. Hear Res. 9, 327 359
Strelioff D and Flock A (1984) Graded and nonlinear mechanical properties of sensory hairs in the mammalian hearing organ. Nature 310, 597-599.

Strelioff D, Flock A, and Minser K E (1985) Role of inner and outer hair cells in mechanical frequency selectivity of the cochlea. Hear Res. 18, 169-175.

Suga N, Simmons J A, and Jen P H S (2003) Peripheral specialization for fine analysis of Doppler-shifted echoes in the auditory system of the CF-FM bat Pteronotus parnellii. J. Exp. Biol. 90, 2261-2273.

Tilney L G and Saunders J C (1983) Actin filaments, stereocilia, and hair cells of the bird cochlea. I. Length, number, width, and distribution of stereocilia of each hair cell are related to the position of the hair cell on the cochlea. J. Cell Biol. 96, 807-821

Vater M and Lenoir M (1992) Ultrastructure of the horseshoe bat's organ of Corti. I. Scanning electron microscopy. J. Comp. Neurol. 318, 367379.

Vater M and Siefer W (1995) The cochlea of Tadarida brasiliensis: specialized functional organization in a generalized bat. Hear Res. 91, 178-195.

Wright A (1984) Dimensions of the cochlear stereocilia in man and the guinea pig. Hear Res. 13, 89-98.

Yao Q, Zheng Y, Latham J, and Zhang S (2007) Characteristics of echolocating bats' auditory stereocilia length, compared with other mammals. Sci. China C. Life Sci. 50, 492-496.

Zenner H P (1986) Motile responses in outer hair cells. Hear Res. 22, 8390. 